DOI: $10.18554 /$ reas.v8i2.3523

\title{
EVIDÊNCIA CIENTÍFICA DOS CUIDADOS DE ENFERMAGEM FRENTE AO PACIENTE COM CARCINOMA ORAL
}

\section{SCIENTIFIC EVIDENCE OF NURSING CARE FOR THE PATIENT WITH ORAL CARCINOMA}

\section{EVIDENCIA CIENTIFICA DE LOS CUIDADOS DE ENFERMATE FRENTE AL PACIENTE CON CARCINOMA ORAL}

\author{
Erlivânia Aparecida de Lucena ${ }^{1}$, Vanessa Mandu Pereira ${ }^{2}$, Kazia Lena Martinele Lopes da \\ Silva Rodrigues ${ }^{3}$, Jackson Jhonatan Vieira do Nascimento ${ }^{4}$, Natália Fonseca de Araújo ${ }^{5}$, \\ Luiza de Marilac Alves da Fonsêca ${ }^{6}$, Vinicius Lino de Souza Neto ${ }^{7}$
}

\section{RESUMO}

Objetivo: Identificar as evidências científicas dos cuidados de enfermagem frente ao paciente com carcinoma oral. Método: Trata-se de uma revisão sistemática, em que se buscou por estudos nas bases de dados Pubmed, Scopus, Cinahl, Web of Science, e The Cochrane Data Base, sobre pesquisas voltadas aos cuidados primários para prevenção do carcinoma oral. Os estudos foram analisados de forma pareada e classificada a sua melhor evidência científica. Resultados: A partir da análise dos 10 estudos foi evidenciado que alguns cuidados de enfermagem apresentam resultados significativos, diante do quadro de dor, prevenção de infecção, e o autocuidado, como o autoexame da orofaríngea e adjacências, que deve ser realizado continuadamente, higiene oral com soro fisiológico e clorexidina, a Crioterapia e o uso do chá de camomila. Conclusão: Os cuidados de enfermagem baseados em estudos científicos contribuem para um maior aprimoramento do processo do trabalho, diminuindo riscos e estimulando habilidades clínicas e acadêmicas.

Descritores: Carcinoma; Enfermagem baseada em evidências; Cuidados de Enfermagem.

\footnotetext{
1 Discente, Graduação em Enfermagem, Faculdade Natalense de Ensino e Cultura de Natal/ FANEC. Natal, Rio Grande do Norte (RN), Brasil. Ciências da Saúde/Enfermagem baseado em envidência

2 Discente, Graduação em Enfermagem, Faculdade Natalense de Ensino e Cultura de Natal/ FANEC. Natal, Rio Grande do Norte (RN), Brasil. Ciências da Saúde/Enfermagem baseado em envidência

3 Discente, Graduação em Enfermagem, Faculdade Natalense de Ensino e Cultura de Natal/ FANEC. Natal, Rio Grande do Norte (RN), Brasil. Ciências da Saúde/Enfermagem baseado em envidência

4 Discente, Graduação em Enfermagem, Universidade Potiguar, UNP, Natal, Rio Grande do Norte (RN), Brasil. Ciências da Saúde/Enfermagem baseado em envidência

5 Enfermeira, graduada pela Universidade Estadual da Paraíba. Especialista em Saúde da Família pela Faculdade Integrada de Patos (FIP). Ciências da Saúde/Enfermagem baseado em envidência

6 Enfermeira, Graduada em Enfermagem pela Universidade Estadual do Rio Grande do Norte- UERN; Mestre em Saúde Coletiva pela Universidade Federal do Rio Grande do Norte - UFRN, Natal, Brasil.

7 Enfermeiro, Graduado pela Universidade Federal de Campina Grande/ UFCG Campina Grande (PB), Mestre pelo Programa de Pós-Graduação em Enfermagem da Universidade federal do Rio Grande do Norte- UFRN.
} 


\begin{abstract}
Objective: To identify the scientific evidences of the nursing care in front of the patient with oral carcinoma. Methods: This is a Systematic review, where studies were conducted in the Pubmed, Scopus, Cinahl, Web of Science, and Cochrane databases for primary care research to prevent oral carcinoma. The studies were analyzed in paired form and classified their best scientific evidence. Results: Based on the analysis of the 10 studies, it was evidenced that some nursing care presents significant results, in view of pain, infection prevention and self-care, such as the self-examination of the oropharyngeal and adjacent ones, which must be performed continuously, oral hygiene with serum physiological and chlorhexidine, cryotherapy and the use of chamomile tea. Conclusion: Nursing care based on scientific studies contributes to further improvement of the work process, reducing risks, and stimulating clinical and academic skills.
\end{abstract}

Descriptors: Carcinoma; Evidence-based nursing; Nursing care.

\title{
RESUMEM
}

Objetivo: Identificar las evidencias científicas de los cuidados de enfermería frente al paciente con carcinoma oral. El método consiste en una revisión sistemática, en la que se buscó estudios en las bases de datos Pubmed, Scopus, Cinahl, Web of Science, y Cochrane, por investigaciones dirigidas a los cuidados primarios para la prevención del carcinoma oral. Los estudios se analizaron de forma pareada y clasificaron su mejor evidencia científica. Resultados: A partir del análisis de los 10 estudios se evidenció que algunos cuidados de enfermería presentan resultados significativos, frente al cuadro de dolor, prevención de infección y el autocuidado, como el autoexamen de la orofaríngea y adyacencias, que debe ser realizada continuadamente, Higiene oral con suero fisiológico y clorexidina, la crioterapia y el uso del té de manzanilla. Conclusión: Los cuidados de enfermería basados en estudios científicos contribuyen a un mayor perfeccionamiento del proceso del trabajo, disminuyendo riesgos, y estimulando habilidades clínicas y académicas.

Descriptores: El carcinoma; Enfermería basada en evidencias; Cuidados de Enfermería

\section{INTRODUÇÃO}

O carcinoma oral é uma afecção neoplásica que pode ser classificada por carcinoma epidermoide, escamocelular e espinocelular, lesões malignas que ficam localizadas na região da língua ou borda lateral posterior. Caracteriza-se por ser um câncer agressivo com altas taxas de invasão local e alto nível metastático. ${ }^{1}$

Assim, entre os principais fatores de risco, como o uso do tabaco, a má higienização, está o Papiloma Virus
Humano (HPV), que é um fator etiológico presente nas análises anátomo-patológicas. Acredita-se que o paciente com câncer requer uma assistência à saúde de forma multidimensional, com ênfase nas suas necessidades prioritárias, requerendo, assim, determinadas habilidade e competências dos profissionais de saúde. ${ }^{2}$

A Enfermagem vem modificando a sua prática, inseridos novos dispositivos e descontruindo o seu modelo empírico, para 
cada vez mais científico, como o cuidado baseado na melhor evidência científica, que requer uma tomada decisão da equipe assistencial. É factível que a assistência baseada em evidência científica ao paciente com carcinoma oral contribui com um cuidado mais qualificado e acurado. ${ }^{3}$

A evidência científica é caracterizada por ser um conjunto de elementos utilizado para apoiar uma teoria científica, que pode variar de acordo com o seu campo de pesquisa. Desta forma, uma evidência geralmente baseia-se em um resultado analítico e controle científico, que, assim, estimula os profissionais a pensar criticamente, e distanciando-os do empirismo. $^{4}$

Diante disso, o Enfermeiro, pautado neste modelo alternativo de assistência à saúde, vigilância em saúde, deve planejar e dimensionar as possíveis necessidade de tal população, por meio de condutas sistemáticas e científicas. ${ }^{5}$ Tornando-o, assim, o cuidado mais evidente e menos empírico, corroborando para o construto da arte e o seu avançar.

Nesse sentido, o desenvolvimento do estudo pauta-se em evidenciar por meio da análise de estudos os cuidados de enfermagem mais apropriados ao paciente com carcinoma oral. Assim, o estudo teve como objetivo: Identificar evidências de cuidados de enfermagem frente ao paciente com carcinoma oral.

\section{MÉTODO}

Trata-se de um estudo de revisão sistemática de literatura que seguiu o protocolo proposto pelo Centro Cochrane do Brasil, que é composto pelas seguintes etapas: formulação da pergunta de pesquisa, em que utilizou-se a estratégia PICO; localização e seleção dos estudos; avaliação crítica dos estudos; coleta de dados; análise e apresentação dos dados; e interpretação dos resultados. E seguindo os passos recomendados pela diretriz Preferred Reporting Items for Systematic Reviews and Meta-Analyses - PRISMA. ${ }^{6}$

$\mathrm{Na}$ formulação da pergunta de pesquisa adotou-se a estratégia PICO, que caracteriza-se por quatro componentes importantes para a formulação da questão de pesquisa, sendo P: participante (patient); $\mathrm{I}$ intervenção (intervention); $\mathrm{C}$ : comparação (comparison) e $\mathrm{O}$ : resultado (outcome). ${ }^{7}$

Assim a pergunta que norteou o estudo de revisão sistemática foi: Quais os cuidados de enfermagem que podem ser evidenciados nos respectivos estudos para as pessoas com carcinoma oral?

A coleta de dados ocorreu no segundo semestre de 2018, por dois pesquisadores[,] de forma pareada. Para as bases de dados Pubmed, Scopus, Cinahl, Web of Science, e The Cochrane Data Base, foram utilizados os seguintes descritores em inglês, selecionados na Medical Subject 
Headings (MeSH), sendo estes: Carcinoma

de boca, Enfermagem baseada em

evidências, Cuidados de Enfermagem.

Além disso, foram utilizados os operadores boolianos AND na estratégia de busca em cada base de dados, como demostra o Quadro 1.

Quadro 1- Distribuição dos cruzamentos entre descritores de ciências da saúde e com operadores boolianos, Natal/RN, 2018

\begin{tabular}{|l|l|}
\hline P & "HPV" AND "Verrugas Genitais" AND "Infecções por Papiloma vírus" AND "Carcinoma" \\
AND "Neoplasia Bucal" AND "DST-HPV" AND "Vacina" AND "Infecções Tumorais por \\
Vírus" AND "Cavidade Oral" AND "Saúde do Homem" AND "Imunidade" AND "Saúde do \\
Estudante" AND "Distribuição por Idade e Sexo" AND "Carcinoma In Situ" AND \\
"Epidemiologia Descritiva" AND "Emoção".
\end{tabular}

Fonte: Pubmed, Scopus, Cinahl, Web of Science, e Cochrane

Para a seleção e inclusão dos artigos na revisão sistemática adotaram-se os seguintes critérios de elegibilidade artigos originais provenientes de pesquisa publicados na íntegra, sem limite de idioma, publicados nas bases nacionais e internacionais. Os critérios de exclusão foram: artigos com enfoque em outra população que não fosse de adultos; não provenientes de pesquisas; e que tratassem de outros tipos de afecções Justifica-se a ausência da temporalidade por ser uma temática com abordagem limitada e que está em menor frequência nos trabalhos.
Assim, por meio da estratégia de busca, foram identificados 38 artigos nas bases de dados. Logo em seguida, após a seleção pelos critérios supracitados, dois pesquisadores analisaram os títulos e resumo, com a pretensão de filtrar os estudos que não colaboravam com o objetivo desta pesquisa, e apenas 10 artigos compuseram a amostra final. De posse do banco de artigos pré-selecionados, foi utilizado um instrumento para a coleta de dados com as respectivas informações: autor, tipo de método aplicado, nível de evidência, resultado e 
conclusão/considerações finais, conforme mostra a Figura 1.

Figura 1 - Processo de seleção dos estudos, Natal/RN, 2018
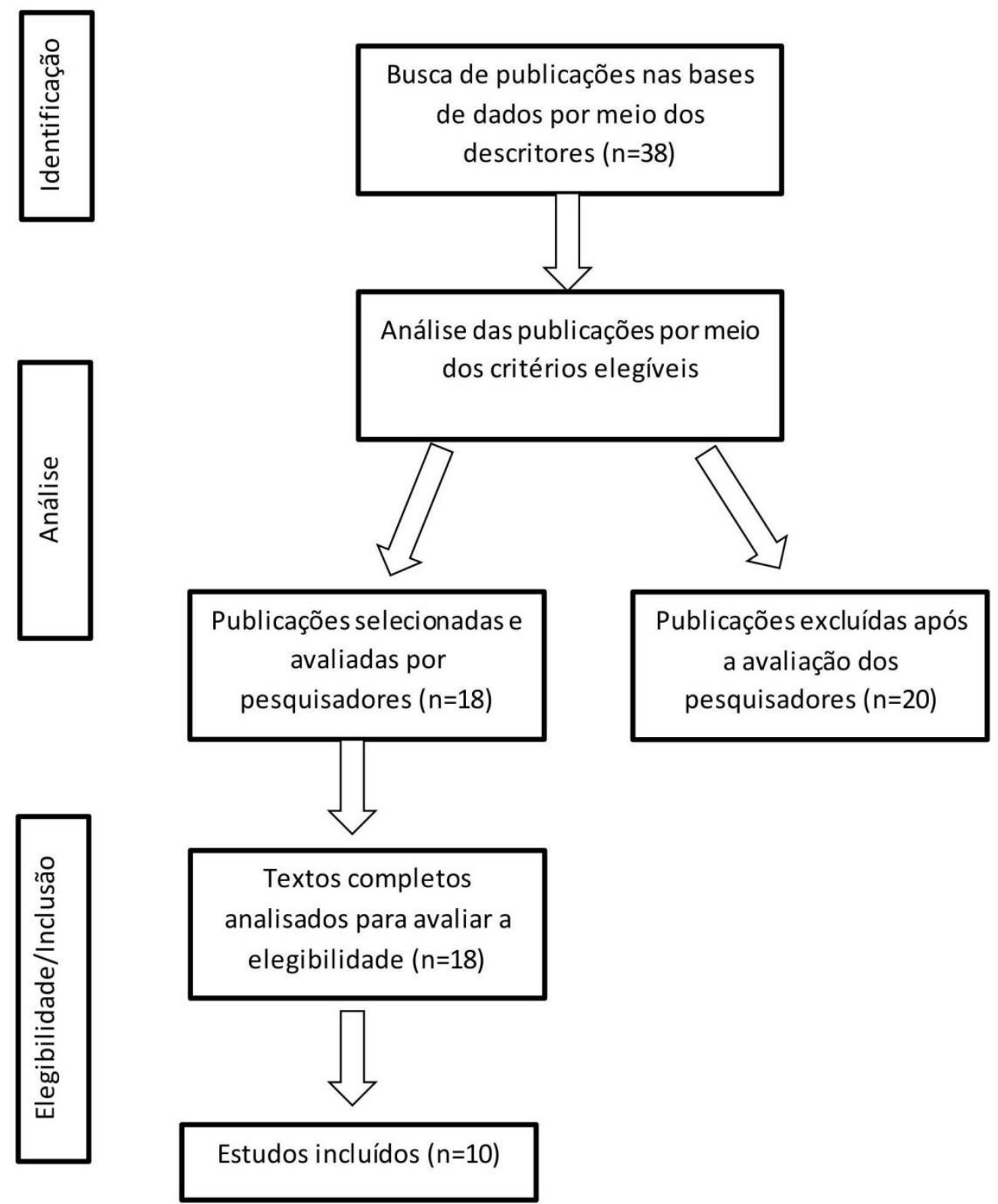

Logo após a catalogação, os estudos passaram por uma análise avaliativa e de qualidade por meio do Strengthening the Reporting of Observational Studies in
Epidemiology (STROBE) para as pesquisas observacionais e do Consolidated Standards of Reporting Trials (CONSORT,) para os estudos de ensaios 
clínicos; e análise do nível de evidência pela classificação do The Joanna Briggs Institute.

Em relação à análise pelo STROBE, adotou-se que cada um dos 22 critérios obtiveram obteria uma pontuação de 0 descrito e 1 - não descrito. Para o CONSORT, que é composto por 22 itens, a pontuação colocada foi 0 - não descrito, 1 descrito parcialmente e adequado e 2 adequado $^{8}$. Assim, a pontuação gerada por artigo foi transformada em percentual, e consideraram-se de qualidade aqueles que apresentavam um percentual maior que
$60 \%$, ou contemplavam características relevantes nesse processo avaliativo. Para avaliar o nível de evidência, para a classificação adotou-se o The Joanna Briggs Institute. ${ }^{8-9}$

\section{RESULTADOS}

Nesta etapa, da amostra de artigos selecionados, foram publicados: um no ano de 2008, um no ano de 2011, um no ano de 2013, dois no ano de 2014, três no ano 2015, dois em 2016. O Quadro 2 sintetiza as informações disponibilizadas pelos artigos que foram incluídos na revisão sistemática.

Quadro 2 - Relação dos artigos que apresentam as ações terapêuticas para o paciente com carcinoma oral, Natal/RN, 2018

\begin{tabular}{|c|c|c|c|}
\hline Fonte & Método/NE* & Resultados & Considerações/Conclusão \\
\hline $\begin{array}{l}\text { Nascimento } \\
\text { DM, Nóbrega } \\
\text { MML, } \\
\text { Carvalho } \\
\text { MWC, Norat } \\
\text { EM }\end{array}$ & $\begin{array}{l}\text { Exploratório- } \\
\text { descritivo/III }\end{array}$ & $\begin{array}{l}\text { Foram elaboradas } 97 \\
\text { afirmativas } \\
\text { diagnósticos/resultados de } \\
\text { enfermagem, que foram } \\
\text { submetidas ao processo de } \\
\text { validação por enfermeiros, } \\
\text { obtendo como resultado } 38 \\
\text { afirmativas com IC } \geq 0.80 \\
(39,2 \%) \text {. }\end{array}$ & $\begin{array}{l}\text { Acredita-se que os diagnósticos de } \\
\text { enfermagem retratam as reais } \\
\text { necessidades dos pacientes } \\
\text { hospitalizados, e fornecem ao } \\
\text { enfermeiro subsídios para identificar as } \\
\text { intervenções de enfermagem } \\
\text { necessárias à assistência desta clientela. }\end{array}$ \\
\hline $\begin{array}{l}02-\text { Araújo } \\
\text { SNM, Luz } \\
\text { MHBA, Silva } \\
\text { GRF, Andrade } \\
\text { EMLR, Nunes } \\
\text { LCC, Moura } \\
\text { RO. }\end{array}$ & $\begin{array}{l}\text { Transversal } \\
\text { quantitativo/IV }\end{array}$ & $\begin{array}{l}\text { A partir disso, delimitou-se } \\
\text { um PE elencando } \\
\text { diagnósticos, intervenções e } \\
\text { resultados esperados, a fim } \\
\text { de se estabelecer um padrão } \\
\text { ideal, porém } \\
\text { individualizante, de } \\
\text { assistência de enfermagem a } \\
\text { estes pacientes. }\end{array}$ & $\begin{array}{l}\text { Conhecer a afecção mucosite oral é } \\
\text { precípuo para formulação de uma } \\
\text { assistência de enfermagem que } \\
\text { vislumbre a prevenção, a partir da } \\
\text { instituição de um plano de cuidados } \\
\text { orais. }\end{array}$ \\
\hline $\begin{array}{l}\text { 03-Santos } \\
\text { NAR, Santos } \\
\text { ATC, Silva RP }\end{array}$ & Qualitativo/III & $\begin{array}{l}\text { Três grandes impressões } \\
\text { foram encontradas: } \\
\text { estranhamento inicial e } \\
\text { complexidade; um limiar } \\
\text { entre estranhamento e } \\
\text { enfrentamento; e imagem. }\end{array}$ & $\begin{array}{l}\text { Entre outras contribuições, a } \\
\text { identificação e compreensão das } \\
\text { estratégias de enfrentamento podem } \\
\text { contribuir para melhor qualificar a } \\
\text { educação e o cuidado em enfermagem. }\end{array}$ \\
\hline $\begin{array}{l}\text { 04-Chedid } \\
\text { HM, Franzi } \\
\text { SA, Amar A, }\end{array}$ & Retrospectivo/III & $\begin{array}{l}\text { Na avaliação da sobrevida } \\
\text { livre de doença de um ano } \\
\text { dos pacientes submetidos à }\end{array}$ & $\begin{array}{l}\text { O tratamento quimiorradioterápico de } \\
\text { resgate nas recidivas locorregionais } \\
\text { apresenta resultados pobres em relação }\end{array}$ \\
\hline
\end{tabular}




\begin{tabular}{|c|c|c|c|}
\hline $\begin{array}{l}\text { Rapoport A, } \\
\text { Dedivitis RA, } \\
\text { Carvalho MB. }\end{array}$ & & $\begin{array}{l}\text { quimiorradioterapia de } \\
\text { resgate, } 16,7 \% \text { estavam } \\
\text { vivos e sem doença, } \\
\text { enquanto que, no grupo dos } \\
\text { pacientes submetidos à } \\
\text { paliação, a sobrevida média } \\
\text { foi de } 3,9 \text { meses. }\end{array}$ & $\begin{array}{l}\text { à sobrevida livre de doença, todavia, } \\
\text { promissores quando comparados aos } \\
\text { pacientes com indicação de cuidados } \\
\text { paliativos. }\end{array}$ \\
\hline $\begin{array}{l}05 \text {-Santos } \\
\text { MCM, } \\
\text { Raimundo DD, } \\
\text { Soares E, } \\
\text { Guedes MTS }\end{array}$ & $\begin{array}{l}\text { Análise } \\
\text { dados/IV }\end{array}$ & $\begin{array}{l}\text { Mostrou a importância dos } \\
\text { registros para a } \\
\text { comprovação do trabalho da } \\
\text { equipe de enfermagem e a } \\
\text { importância da Clínica } \\
\text { Ampliada como filosofia e } \\
\text { ferramenta para que os } \\
\text { processos de trabalho em } \\
\text { saúde se voltem para a } \\
\text { produção do cuidado } \\
\text { centrado nos clientes e as } \\
\text { contradições na tentativa de } \\
\text { inter-relação com serviços } \\
\text { de saúde fora da instituição. }\end{array}$ & $\begin{array}{l}\text { O atendimento empático, planejado e } \\
\text { coordenado visando à recuperação e } \\
\text { adaptação do cliente mostrou-se } \\
\text { imprescindível e resultou em um plano } \\
\text { de cuidados flexível desenvolvido para } \\
\text { cada pessoa, contendo medidas } \\
\text { educativas e terapêuticas de } \\
\text { enfermagem e de encaminhamento dos } \\
\text { problemas interdisciplinares, } \\
\text { respeitando a individualidade e } \\
\text { priorizando seus problemas. }\end{array}$ \\
\hline $\begin{array}{l}\text { 06-Guimarães } \\
\text { RCR, Fonseca } \\
\text { Gonçalves } \\
\text { RPF, Lima } \\
\text { CA, Rocha } \\
\text { Torres M, } \\
\text { Silva CSO. }\end{array}$ & Integrativa/V & $\begin{array}{l}\text { A náusea e o vômito foram } \\
\text { as principais reações da } \\
\text { quimioterapia descritas. }\end{array}$ & $\begin{array}{l}\text { Constatou-se a necessidade de melhoria } \\
\text { dos registros de enfermagem, bem } \\
\text { como a implantação do processo } \\
\text { avaliativo na construção de indicadores } \\
\text { para avaliar condutas e/ou intervenções } \\
\text { específicas. }\end{array}$ \\
\hline $\begin{array}{l}\text { 07- Araújo SN, } \\
\text { et al. }\end{array}$ & $\begin{array}{l}\text { Exploratório- } \\
\text { descritivo/IV }\end{array}$ & $\begin{array}{l}\text { Os achados apontaram a } \\
\text { prevalência de mucosite oral } \\
\text { no sexo masculino e nas } \\
\text { faixas etárias inferiores a } 17 \\
\text { e superiores a } 60 \text { anos. }\end{array}$ & $\begin{array}{l}\text { Conclui-se que há necessidade da } \\
\text { inserção da enfermagem no fomento às } \\
\text { ações preventivas e de controle da } \\
\text { mucosite oral, com vistas à manutenção } \\
\text { do bem-estar, otimização da resposta } \\
\text { terapêutica e melhoria da qualidade de } \\
\text { vida do paciente oncológico. }\end{array}$ \\
\hline $\begin{array}{l}\text { 08-Moura } \\
\text { LKB }\end{array}$ & $\begin{array}{l}\text { Revisão } \\
\text { integrativa/V }\end{array}$ & $\begin{array}{l}\text { O rastreio do câncer oral } \\
\text { para diminuição } \\
\text { prevalência. }\end{array}$ & $\begin{array}{l}\text { O câncer oral é um grave problema de } \\
\text { saúde pública no Brasil e no mundo. }\end{array}$ \\
\hline $\begin{array}{l}\text { 09-Raimundo } \\
\text { DD et al. }\end{array}$ & $\begin{array}{l}\text { Retrospectivo- } \\
\text { analítico/IV }\end{array}$ & $\begin{array}{lr}\text { Os principais } & \text { DEs } \\
\text { identificados } & \text { foram } \\
\text { deglutição prejudicada, dor, } \\
\text { comunicação } & \text { verbal } \\
\text { prejudicada relacionados ao } \\
\text { tumor; processo familiar } \\
\text { disfuncional por álcool e } \\
\text { tabaco, tensão do papel de } \\
\text { cuidador, risco de baixa } \\
\text { autoestima[,] entre outros. }\end{array}$ & $\begin{array}{l}\text { Registro acurado sobre paciente e } \\
\text { cuidador pode minimizar sofrimento. }\end{array}$ \\
\hline 10- Ribeiro JP & Quantitativo/IV & $\begin{array}{l}\text { O diagnóstico identificado } \\
\text { concentra-se nos domínios } \\
\text { da Autopercepção, papéis e } \\
\text { relacionamento, confronto } \\
\text { total ao estresse, conforto e } \\
\text { Princípios da vida. }\end{array}$ & $\begin{array}{l}\text { É imprescindível entender o impacto } \\
\text { causado pelo câncer, pois tal } \\
\text { entendimento possibilita } \\
\text { estabelecimento de diagnósticos, bem } \\
\text { como intervenções de enfermagem } \\
\text { adequadas no momento vivenciado. }\end{array}$ \\
\hline
\end{tabular}

Fonte: Pubmed, Scopus, Cinahl, Web of Science, e The Cochrane Data Base; NE*- Nível de evidência.

Os estudos evidenciaram que os cuidados de enfermagem com melhor resultado para o paciente com carcinoma oral foram: o autoexame da orofaríngea e 
adjacências, que deve ser realizado continuadamente, higiene oral com soro fisiológico e clorexidina, a Crioterapia e o uso do chá de camomila.

\section{DISCUSSÃO}

A assistência à saúde desse paciente deve ser de forma holística e multidisciplinar, requerendo determinadas habilidades e competências dos profissionais. ${ }^{10-12}$ Porém, apesar do aumento de conhecimento científico sobre o carcinoma oral, a Enfermagem apresenta fragilidades nessa área, como, por exemplo, a comunicação das ações de prevenção, promoção, proteção, e curativas e de reabilitação desses pacientes.

Assim, dentre as diversas alternativas terapêuticas, os estudos com níveis de evidência III e IV apontam que a prática do autoexame oral, seja de forma visual ou tátil, tem como finalidade identificar anormalidades na cavidade oral, como lesões persistentes, sangramentos na gengiva, endurecimento na língua. Além disso, devem-se realizar exames complementares como palpação submandibular, submentonianas e cervicais, podendo ser notificados linfonodos aumentados. ${ }^{13-15}$

Já, em outros estudos com nível de evidência II e III, a alternativa implementada foi a higienização oral preventiva por digluconato de clorexidina a
$0,12 \%$, pois o produto apresenta ações enzimáticas, impedindo as células da proliferação da afecção. ${ }^{16-19}$ Ainda assim, estudos comprovam que o uso prolongado do digluconato de clorexidina a $0,12 \%$ resulta em efeitos adversos, podendo causar alterações nos dentes, aumentando a calcificação supragengiva, pois a clorexidina a $0,12 \%$ não previne a mucosite, mas proporciona conforto aos clientes.

Além da clorexidina $0,12 \%$, o uso da solução salina a $0,9 \%$ ou bicarbonatada a 3\% não irrita a mucosa oral e possui a ação de modificação do $\mathrm{pH}$ salivar, conforme aponta estudo desenvolvido pela cidade do México. Em que se comparou o bochecho diário do digluconato de clorexidina com o uso da solução, o que evidenciou benefício dos dois lados e que a as duas substâncias apresentam desconforto para o paciente, como a mudança do paladar. Outro ponto evidenciado no estudo foi que a frequência do uso deve ser de seis vezes ao dia. ${ }^{18}$

Outra modalidade terapêutica que vem ganhando destaque é o uso do laser e da crioterapia. Estudos com níveis de evidência III e IV mostram que o uso da crioterapia, que se caracteriza pela diminuição da temperatura, proporcionando a ativação dos receptores adrenérgicos, que assim leva à vasoconstricção e a diminuir a liberação de citoquinas, que são substâncias que aumentam o processo de proliferação 
oncológica, vem sendo implementado como medida terapêutica, seja para o alívio da dor, ou para diminuição do processo infecioso do carcinoma oral. ${ }^{19-20}$

Além disso, a crioterapia vem sendo bastante utilizada em outras vertentes terapêuticas, como aponta a Multinational Association of Supportive Care in Cancer and International Society of Oral Oncology, que coloca nas suas diretrizes o uso da crio pelo menos duas a três vezes ao dia, conforme a necessidade do paciente e a acessibilidade de uso. Outra terapêutica evidenciada pelo estudo de nível II, e que está ainda na busca por resultados amplos, é o uso do chá de camomila, que proporciona a diminuição do processo de catabolização das células neoplásicas, inibindo determinados tipos de enzimas oncogênicas que levam à diminuição de substâncias que acarretam odores. ${ }^{20}$

\section{CONCLUSÃO}

Os cuidados de enfermagem que vêm sendo implementados junto com estratégias sistemáticas, baseados em estudos científicos, contribuem para um maior aprimoramento do processo do trabalho, otimizado tempo, diminuindo riscos e incentivando reflexões, e estimulando habilidades clínicas e acadêmicas da equipe de enfermagem.

No presente estudo, os cuidados de enfermagem ao paciente com carcinoma oral que foram mais evidentes são: o autoexame da orofaríngea e adjacências, que deve ser realizado continuadamente, higiene oral com soro fisiológico e clorexidina, a Crioterapia e o uso do chá de camomila.

As limitações do estudo pautam-se no maior dimensionamento de pesquisadores da área de enfermagem com a temática, pois ainda é pouco explorada, seja por pesquisadores nacionais, ou internacionais. Porém, acredita-se que tal estudo promova novos caminhos em relação à temática e que tais cuidados possam ser instituídos no processo de trabalho.

\section{REFERÊNCIAS}

1.Nicolatou-Galitis O, Sarri T, Bowen J, Di Palma M, Kouloulias VE, Niscola P, et al. Systematic review of anti-inflammatory agents for the management of oral mucositis in cancer patients. Support Care Cancer. 2013; 21(11):3179-89.

2.Lalla RV, Saunders DP, Peterson DE. Chemotherapy or radiation-induced oral mucositis. Dent Clin North Am. 2014; 58(2):341-9.

3.Assis YMS, Alves KYA, Santos VEP. Evidências científicas do cuidado de enfermagem e segurança do paciente em unidade de internação oncológica. Rev Cuba Enferm. 2016; 32(3):442-58.

4.McCrae N. Whither nursing models? The value of nursing theory in the context of evidence-based practice and multidisciplinary health care. J Adv Nurs. 2012; 68(1):222-9. 
5.Beyea SC, Slattery MJ. Historical perspectives on evidence-based nursing. Nurs Sci Q. 2013; 26(2):152-5.

6. Moher D, Liberati A, Tetzlaff J, Altman DG. Preferred reporting items for systematic reviews and meta-analyses: the PRISMA statement. PloS Med. [Internet]. 2009 [citado em 24 jan 2019]; 6(7):e1000097. Disponível em: https://journals.plos.org/plosmedicine/artic le? id=10.1371/journal.pmed.1000097 1599

7.Spolarich AE. Risk management strategies for reducing oral adverse drug events. J Evid Based Dent Pract. 2014; 14(Suppl):87-94.

8.Lopes LD, Rodrigues AB, Brasil DRM, Moreira MMC, Amaral JG, Oliveira PP. Prevenção e tratamento da mucosite em ambulatório de oncologia: uma construção coletiva. Texto \& Contexto Enferm. 2016; 25(1):45-9.

9.Peterson DE, Ohrn K, Bowen J, Fliedner M, Lees J, Loprinzi C, et al. Systematic review of oral cryotherapy for management of oral mucositis caused by cancer therapy. Support Care Cancer. 2013; 21(1):327-32

10.Brito CA, Araújo DS, Granja JG, Souza SM, Lima MAG, Oliveira MC. Efeito da clorexidina e do laser de baixa potência na prevenção e no tratamento da mucosite oral. Rev Odontol UNESP. 2012;

41(4):236-41

11.Nascimento DM, Nóbrega MML, Carvalho MWA, Norat EM. Diagnósticos, resultados e intervenções de enfermagem para clientes hospitalizados submetidos à prostatectomia Rev Eletrônica Enferm. [Internet]. 2011[citado em 24 jan 2019];13(2):165-73. Disponível em: https://www.revistas.ufg.br/fen/article/vie w/11117

12.Araújo SNM, Luz MHBA, Silva GRF, Andrade EMLR, Nunes LCC, Moura RO. O paciente oncológico com mucosite oral: desafios para o cuidado de enfermagem. Rev Latinoam Enferm. 2015; 23(2):267-74 13.Santos NAR, Santos ATC, Silva RP. Coping strategies of nurses in the care of patients with head and neck neoplasms.

Rev Esc Enferm USP. 2016; 50(4):569-78. 14.Chedid HM, Franzi AS, Amar A, Rapoport A, Dedivitis RA, de Carvalho MB. Mudança do paradigma no carcinoma espinocelular recidivado de boca e orofaringe: paliação versus quimioradioterapia. Rev Bras Cir Cabeça Pescoço. 2008; 37(1):32-6.

15.Santos MCM, Raimundo DD, Soares E, Santos Guedes MT. Assistência aos portadores de câncer de laringe sob a perspectiva da integralidade: abordagem do enfermeiro no Inca. Rev Pesqui Cuid Fundam. [Internet]. 2015 [citado em 24 jan 2019]; 7(3):2649-658. Disponível em: http://www.seer.unirio.br/index.php/cuidad ofundamental/article/view/3639/pdf_1599 16.Guimarães RCR, Gonçalves RPF, Lima CA, Torres MR, Oliveira e Silva CS. Ações de enfermagem frente às reações a quimioterápicos em pacientes oncológicos. Rev Pesqui Cuid Fundam. [Internet]. 2015 [citado em 24 jan 2019]; 7(2):2440-452. Disponível em:

http://www.seer.unirio.br/index.php/cuidad ofundamental/article/view/3589/pdf_1559 17.Araújo SN, Luz MHBA, de Almeida LHRB, Silva GRF, Neto JMM, Costa ACMMA. Oncological patients and the nursing field: ration between the oral mucositis grade and the implemented therapeutic. Rev Pesqui Cuid Fundam. [Internet]. 2013[citado em 24 jan 2019]; 5(4):386-95. Disponível em: http://www.seer.unirio.br/index.php/cuidad ofundamental/article/view/2624/pdf_891 18. Moura LKB, Marcaccini AM, Matos FTC, de Sousa ÁFL, Nascimento GC, Moura MEB. Revisão integrativa sobre o câncer bucal. Rev Pesqui Cuid Fundam. [Internet]. 2014 [citado em 24 jan 2019]; 6(5):164-75. Disponível em:

http://www.seer.unirio.br/index.php/cuidad ofundamental/article/view/4516/pdf_1511 19. Raimundo DD, Guedes MTS, Luzial NS, Peixoto MGS, Santos MCM, Silva CC. Assistência de enfermagem a clientes com câncer na cabeça e no pescoço com ênfase nos tumores de cavidade oral no 
estado do Rio de Janeiro. Rev Pesqui Cuid Fundam. [Internet]. 2014 [citado em 24 jan 20190]; 6(4):1496-504. Disponível em:

http://www.seer.unirio.br/index.php/cuidad ofundamental/article/view/2088/pdf_979 20.Ribeiro JP, Cardoso LS, Pereira CMS, Silva BT, Bubolz BK, Castro CK.

Assistência de enfermagem ao paciente oncológico hospitalizado: diagnósticos e intervenções relacionadas às necessidades psicossociais e psicoespirituais. Rev Pesqui Cuid Fundam. [Internet]. 2016 [citado em 24 jan 2019]; 8(4):5136-142. Disponível em:

http://www.seer.unirio.br/index.php/cuidad ofundamental/article/view/4016/pdf_1
RECEBIDO: 17/01/2019

APROVADO: $11 / 07 / 2019$

PUBLICADO: 12/2019 